Pacific Journal of Mathematics

ON SPECTRAL THEORY AND SCATTERING FOR ELLIPTIC 


\title{
ON SPECTRAL THEORY AND SCATTERING FOR ELLIPTIC OPERATORS WITH SINGULAR POTENTIALS
}

\section{RICHARD BEALS}

\begin{abstract}
General conditions have been found which imply that the perturbation $A+q$ of an elliptic differential operator $A$ by a singular potential term $q(x)$ has a closed extension $B$ in $L^{2}\left(R^{n}\right)$ having the same essential spectrum as $A$. The purpose of this paper is to sharpen the known results slightly and to estimate the characteristic numbers of the operator $(A+\lambda)^{p}-(B+\lambda)^{p}$. Under an appropriate assumption on $q(x)$, this operator is shown to be of trace class for large $p$. In the self-adjoint case it follows then from results of Kato that wave operators for the pair $(A, B)$ exist and that the absolutely continuous parts of these operators are unitarily equivalent.
\end{abstract}

Let $r$ be a positive integer and let

$$
A(x, D)=\sum_{|\alpha| \leqq r,|\beta| \leqq r} D^{\alpha}\left(a_{\alpha \beta}(x) D^{\beta}\right)
$$

be a differential operator of order $m=2 r$. Here

$$
x \in R^{n}, \alpha=\left(\alpha_{1}, \cdots, \alpha_{n}\right),|\alpha|=\sum \alpha_{j},
$$

and $D^{\alpha}=(-i)^{|\alpha|} \Pi\left(\partial / \partial x_{j}\right)^{\alpha_{j}}$. We assume throughout that $a_{\alpha \beta}$ has continuous derivatives of order $\leqq \max \{|\alpha|,|\beta|\}$, and the derivatives are uniformly bounded. For $|\alpha|=|\beta|=r$ we assume $a_{\alpha \beta}$ uniformly continuous. Finally, $A(x, D)$ is uniformly strongly elliptic: there is a constant $a_{0}>0$ such that

$$
\operatorname{Re}\left(\sum_{|\alpha|=|\beta|=r} a_{\alpha \beta}(x) \xi^{\alpha} \xi^{\beta}\right) \geqq a_{0}|\xi|^{m}
$$

for all $x \in R^{n}, \xi \in R^{n}$.

Let $A_{0}$ be the restriction of $A(x, D)$ to $\mathscr{D}$, the smooth functions with compact support. Let $A$ be the closure of $A_{0}$ in $L^{2}=L^{2}\left(R^{n}\right)$. Various conditions have been given on a potential term $q(x)$ such that $A+q$ have a closed extension $B$ with the same essential spectrum as $A$, either generally or in the particular case $A=-\Delta$; [1], [2], [4], [6]. The most general result of this sort seems to be that of Schechter [8], [9]. We shall sharpen Schechter's result and then investigate the characteristic numbers of $(A+\lambda)^{-p}-(B+\lambda)^{-p}$.

For $\mu>-n$ and $x \neq 0$, set $\omega_{\mu}(x)=|x|^{\mu}$ if $\mu<0$, 


$$
\omega_{0}(x)=(-\log |x|)^{+}, \omega_{\mu}(x)=1
$$

if $\mu>0$. Suppose $q$ is a measurable function defined on $R^{n}$ and suppose $\mu>-n, p>0, \delta>0, x \in R^{n}$. Let

$$
\begin{aligned}
M_{\mu, p, \delta, x}(q) & =\int_{|y|<\delta}|q(x-y)|^{p} \omega_{\mu}(y) d y \\
M_{\mu, p, x}(q) & =M_{\mu, p, 1, x}(q) \\
M_{\mu, p, \delta}(q) & =\sup _{x} M_{\mu, p, \delta, x}(q) \\
M_{\mu, p}(q) & =M_{\mu, p, 1}(q) .
\end{aligned}
$$

We shall assume throughout that

$$
\begin{aligned}
& M_{2 m-n, 1}(q)<\infty, M_{m-n, 1}(\operatorname{Im} q)<\infty, \text { and } \\
& M_{m-n, 1, \delta}\left((\operatorname{Re} q)^{-}\right) \rightarrow 0 \text { as } \delta \rightarrow 0, \text { if } m \leqq n .
\end{aligned}
$$

Note that these conditions are implied by

$(\mathrm{I})^{\prime}$.

$$
\begin{aligned}
& M_{2 m-n, 1}\left((\operatorname{Re} q)^{+}\right)<\infty, M_{m-n, 1}(\operatorname{Im} q)<\infty, \\
& M_{\mu, 1}\left((\operatorname{Re} q)^{-}\right)<\infty \text { for some } \mu<m-n .
\end{aligned}
$$

In fact $M_{\mu, p}(q) \leqq c(\mu, \nu, \delta) M_{\nu, p, o}(q)$ if $\mu \leqq \nu$. Thus the conditions (I)' imply the first two conditions in (I). If $m \leqq n$, take $\mu<\nu<m-n$. The Schwarz inequality gives

$$
\begin{aligned}
{\left[M_{\nu, 1, \delta, x}(q)\right]^{2} } & \leqq M_{\mu, 1, \dot{\delta}, x}(q) \int_{|y|<\delta}|q(x-y)||y|^{2 \nu-\mu} d y \\
& \leqq\left[M_{\mu, 1, \delta}(q)\right]^{2} \delta^{2 \nu-2 \mu},
\end{aligned}
$$

which implies the third condition in (I). Note that if $m>n$ then (I) and (I)' coincide; in fact $M_{\nu, p}(f)$ is constant for $\nu>0$.

Take measurable functions $\rho, \sigma, \tau$ such that

$$
\rho^{2}=(\operatorname{Re} q)^{+}, \sigma^{2}=(\operatorname{Re} q)^{-}, \tau^{2}=\operatorname{Im} q .
$$

For real $l$ let

$$
\|u\|_{l}^{2}=\int\left(1+|\xi|^{2}\right)^{l}|\widehat{u}(\xi)|^{2} d \xi, u \in \mathscr{D},
$$

where $u$ denotes the Fourier transform of $u$. Let $H^{l}$ be the completion of $\mathscr{D}$ with respect to this norm. Then $H^{0}=L^{2}$. When $l$ is a nonnegative integer, an equivalent norm is

$$
\sum_{\mid \alpha, \leqq l}\left\|D^{\alpha} u\right\|_{0}^{2} .
$$

Let $W^{r}$ be the completion of $\mathscr{D}$ with respect to the norm 


$$
|u|^{2}=\|u\|_{r}^{2}+\|\rho u\|_{0}^{2}
$$

Let

$$
b(u, v)=a(u, v)+(q u, v)=\sum\left(a_{\alpha \beta} D^{\beta} u, D^{\alpha} v\right)+(q u, v)
$$

for $u, v \in \mathscr{D}$, where $(u, v)$ is the $L^{2}$-inner product. As in [9], this form extends to $W^{r}$. Define $B_{0}$ as an operator in $H^{0}$ by $B_{0} u=A u+q u$ for $u \in \mathscr{D}$ such that $q u \in H^{0}$. Define $B$ by $B u=f$ in $H^{0}$ if and only if $u \in W^{r}$ and $b(u, v)=(f, v)$ for all $v \in W^{r}$.

Theorem 1. $B$ is a closed extension of $B_{0}$. There is a constant $\lambda_{0}$ such that $A+\lambda$ and $B+\lambda$ are $1-1$ and onto for $\operatorname{Re} \lambda \geqq \lambda_{0}$. If $a_{\alpha, 3}=\bar{a}_{\alpha \beta}$ and $q$ is real-valued, then $A$ and $B$ are self-adjoint.

The essential spectrum of a closed operator $T$ in the sense of Schechter [7], $\sigma_{e m}(T)$, is the complement of the set of complex $\lambda$ such that $T-\lambda$ has finite-dimensional null space $N$ and closed range of codimension equal to the dimension of $N$.

\section{THEOREM 2. Suppose}

$$
M_{2 m-n, 1, x}(q) \rightarrow 0 \text { as }|x| \rightarrow \infty .
$$

Then for $\operatorname{Re} \lambda \geqq \lambda_{0},(A+\lambda)^{-1}-(B+\lambda)^{-1}$ is compact. Consequently $A$ and $B$ have the same essential spectrum.

The characteristic numbers of a bounded operator $S$ in a Hilbert space are defined by

$$
\mu_{j}(S)=\inf _{\operatorname{codim}\left(H_{j}\right)<j} \sup _{u \in H_{j},\|u\|=1}\|S u\|, j=1,2, \cdots .
$$

For compact $\mathbb{S}$, these are the eigenvalues of $\left(S^{*} S\right)^{1 / 2}$ arranged in nonincreasing order.

Theorem 3. Suppose $a>0$ and

(III) $)_{\mathrm{a}}$

$$
\begin{gathered}
M_{2 m-n, 1, x}\left((\operatorname{Re} q)^{+}\right)=0\left(|x|^{-2 a}\right) \text { as }|x| \rightarrow \infty, \\
M_{m-n, 1, x}\left(q-(\operatorname{Re} q)^{+}\right)=0\left(|x|^{-a}\right) \text { as }|x| \rightarrow \infty,
\end{gathered}
$$

or

(III) $)_{a}^{\prime}$

$$
M_{m-n, 1, x}(q)=0\left(|x|^{-a}\right) \text { as }|x| \rightarrow \infty .
$$

Then for any $\varepsilon>0$ there is an integer $p(\varepsilon)$ such that

$$
\mu_{j}\left((A+\lambda)^{-p}-(B+\lambda)^{-p}\right)=0\left(j^{s-a / n}\right) \text { as } j \rightarrow \infty
$$

for $p \geqq p^{(}(\varepsilon), \operatorname{Re} \lambda \geqq \lambda_{\lrcorner}$. 
THeOREM 4. Suppose (III) or (III)' holds for some $a>n$, and suppose $A$ and $B$ are self-adjoint. Let $P_{0}$ and $P_{1}$ be the projections on the absolutely continuous subspaces of $A$ and $B$ respectively. Then the wave operators

$$
\begin{gathered}
W_{ \pm}(B, A)=s-\lim _{t \rightarrow \pm \infty} e^{i t B} e^{-i t A} P_{0}, \\
W_{ \pm}(A, b)=s-\lim _{t \rightarrow \pm \infty} e^{i t A} e^{-i t B} P_{1}
\end{gathered}
$$

exist. The operators $A P_{0}$ and $B P_{1}$ are unitarily equivalent.

REMARKS 1. Theorem 1 is proved in [9] under the stronger assumption $(\mathrm{I})^{\prime}$.

2. Theorem 2 is proved in [9] under the assumptions $(\mathrm{I})^{\prime}$ and (II)', where

$$
\begin{gathered}
M_{\nu, 1}\left((\operatorname{Re} q)^{+}\right)<\infty \text { for some } \nu<2 m-n, \\
\int_{|y| \leqq 1}|q(x-y)| d y \rightarrow 0 \text { as }|x| \rightarrow \infty .
\end{gathered}
$$

Again these assumptions imply ours. If $2 m>n$, (II)' is the same as (II). Otherwise take $\nu<\eta<2 m-n$. Then $M_{2 m-n, 1, x}(q) \leqq c M_{\eta, 1, x}(q)$ and Holder's inequality gives

$$
M_{\eta, 1, x}(q) \leqq\left[M_{\eta p, 1, x}(q)\right]^{1 / p}\left[\int_{|y| \leqq 1}(q(x-y)]^{1 / p^{\prime}}\right.
$$

for $1<p<\infty, 1 / p+1 / p^{\prime}=1$. Taking $p=\nu / \eta$ and using (II)', we get (II).

3. Theorems 3 and 4 are not difficult when $r$ is large relative to $n$ or when $q$ is smooth. However in the Schrodinger case $r=1$, and in general one wants to allow singular $q$. Our methods for handing the general case are cumbersome, but effective.

4. There is a large body of literature on the existence of wave operators, but none of the previous work seems to cover explicitly general potentials as locally singular as those considered here.

2. Proof of Theorem 1. The proof is a modification of the proofs of similar theorems in [8] and [9], to which we refer for details.

For $s>0$, let $g_{s}$ be the tempered distribution with Fourier transform $(2 \pi)^{-n / 2}\left(1+|\xi|^{2}\right)^{-s / 2}$. Then $g_{s}$ is in $L^{1}$ and

$$
\begin{aligned}
\left|g_{s}(x)\right| & \leqq c_{s} \omega_{s-n}(x) \text { for }|x| \leqq 1 \\
& \leqq c_{s} \exp \left(-d_{s}|x|\right) \text { for }|x|>1, \text { where } d_{s}>0 .
\end{aligned}
$$


From (2) we get

$$
\|u\|_{t+s}=\left\|G_{s} u\right\|_{t} \text {, all real } t,
$$

where $G_{s} u$ is the convolution $g_{s} * u$. In fact

$$
\left[G_{s} u\right]^{\wedge}=\left(1+|\xi|^{2}\right)^{s / 2} \widehat{u} .
$$

We can use this equation to define $G_{s}$ for $s \leqq 0$; then (6) holds and $G_{s} G_{t}=G_{s+t}$ for all $s, t$.

LEMma 1. If $s>0$ and $M_{2 s-n, 2}(f)<\infty$, then multiplication by $f$ maps $H^{s}$ into $H^{0}$ and $H^{0}$ into $H^{-s}$. Moreover

$$
\begin{gathered}
\|f u\|_{0}^{2} \leqq c M_{2 s-n, 2}(f)\|u\|_{s}^{2}, \\
\|f u\|_{-s}^{2} \leqq c M_{2 s-n, 2}(f)\|u\|_{0}^{2},
\end{gathered}
$$

$c$ independent of $u$ and $f$.

This is proved in [8]. We also need a sharper form.

Lemma 2. If $s>0$ and $M_{2 s-n, 2}(f)<\infty$, then for all $\delta>0$,

$$
\|f u\|_{0}^{2} \leqq c M_{2 s-n, 2, \dot{o}}(f)\|u\|_{s}^{2}+c(\delta) M_{2 s-n, 2}(f)\|u\|_{0}^{2},
$$

with $c$ independent of $f, u$, and $\delta$.

Proof. Choose $\varphi \in \mathscr{D}$ such that $\varphi(x)=1,|x|<1 / 4, \varphi(x)=0$, $|x|>1 / 2$. Let $\varphi_{\hat{o}}(x)=\varphi\left(\delta^{-1} x\right)$. Let $g_{s}=\varphi_{\hat{\delta}} g_{s}+\left(1-\varphi_{\hat{\delta}}\right) g_{s}=k_{\hat{o}}+l_{\hat{\delta}}$. Let $K_{\hat{o}} u=k_{\hat{\delta}}^{*} u, L_{\hat{o}} u=l_{\hat{o}}^{*} u$. Then

$$
\|f u\|_{0}=\left\|f G_{s} G_{-s} u\right\|_{0} \leqq\left\|f K_{\hat{o}} G_{s} u\right\|_{0}+\left\|f L_{\hat{o}} G_{s} u\right\|_{0} \text {. }
$$

We want to compute $\left\|f K_{\delta}\right\|$, the norm as operator in $H^{0}$. But $\left\|f K_{\delta}\right\|^{2}=\left\|f K_{\delta}\left(K_{\delta} f\right)^{*}\right\|=\left\|f K_{\delta} K_{\delta}^{*} f^{*}\right\|$, where $f^{*}$ is the complex conjugate function. Now $K_{\delta} K_{\delta}^{*}$ is convolution with $k=k_{\delta}^{*} k_{\delta}^{*}$ and this is easily shown to have $|k(x)| \leqq \mathrm{c}_{0} \omega_{2 s-n}(x)$ for $|x|<\delta, k(x)=0,|x|>\delta$, with $c_{0}$ independent of $\delta$. Then the Schwarz inequality gives

$$
\begin{aligned}
\left|\left(f k_{\delta} K_{\tilde{o}}^{*} f^{*} u, v\right)\right|^{2} & \leqq \int\left|f(x)^{2} k(x-y) u(y)^{2}\right| d y d x \int\left|f(y)^{2} k(x-y) v(x)^{2}\right| d x d y \\
& \leqq c_{1}^{2}\left[M_{2 s-n, 2, \delta}(f)\right]^{2}\|u\|_{0}^{2}\|v\|_{0}^{2} .
\end{aligned}
$$

This shows that $\left\|f K_{\delta}\right\|^{2} \leqq c_{1} M_{2 s-n, 2, \delta}(f)$. Finally, $l_{\hat{o}}$ is a smooth function of rapid decrease, so $L_{\delta}$ is continuous from $H^{0}$ to $H^{s}$. Combining these facts with (6), (7), and (10) we get (9).

Our assumptions on $A(x, D)$ imply [8]

$$
\operatorname{Re} a(u, u)=\operatorname{Re}\left(A_{0} u, u\right) \geqq c_{1}\|u\|_{r}^{2}-c_{2}\|u\|_{r}^{2}, u \in \mathscr{D},
$$


where $c_{1}>0$. Conversely it is clear that

$$
|a(u, v)| \leqq c_{3}\|u\|_{r}\|v\|_{r}, u, v \in \mathscr{D} .
$$

From (12), (7), (3) and assumption (I) we get

$$
|b(u, v)| \leqq c_{4}|u||v|, u, v \in \mathscr{D} .
$$

Therefore $b(u, v)$ extends uniquely to a continuous form on $W^{r}$. When $m<n$, assumption (I) and (9), (11) give

$$
\begin{aligned}
\operatorname{Re} b(u, u) & =\operatorname{Re} a(u, u)+\|\rho u\|_{0}^{2}-\|\sigma u\|_{0}^{2} \\
& \geqq c_{5}|u|^{2}-c_{6}\|u\|_{0}^{2},
\end{aligned}
$$

with $c_{5}>0$. This also holds when $m \geqq n$; here multiplication by $\sigma$ is continuous from $H^{s}$ to $H^{0}$ for any $s>0$. Taking $0<s<r$ and using the well-known fact that for $\varepsilon>0$,

$$
\|u\|_{s}^{2} \leqq \varepsilon\|u\|_{r}^{2}+c(\varepsilon)\|u\|_{0}^{2},
$$

we again can get (14).

It is now easy to show that $B$ as defined in $\S 1$ is a closed extension of $B_{0}$, cf. [9]. The fact that $B+\lambda$ is $1-1$ and onto for Re $\lambda \geqq \lambda_{0}>c_{6}$ follows from (14) and the Lax-Milgram lemma as in [9]. Finally, if $a_{\alpha \beta}=\bar{a}_{\alpha \beta}$ and $q$ is real-valued, then for $u, v$ in the domain of $B$,

$$
(B u, v)=b(u, v)=b(v, u)^{*}=(B v, u)^{*}=(u, B v) .
$$

Thus $B$ is symmetric. For $\lambda \geqq \lambda_{0},(B+\lambda)^{-1}$ is symmetric, hence self-adjoint. Then $B+\lambda$ and $B$ are self-adjoint. It can be shown that $A$ as defined above is the same as $A$ defined by $A u=f \in H^{0}$ if and only if $u \in H^{r}$ and $a(u, v)=(f, v)$, all $v \in H^{r}$. Then the same results hold for $A$.

3. Proof of Theorem 2. Suppose $\operatorname{Re} \lambda \geqq \lambda_{0}$. For convenience we may replace $A, B$ by $A+\lambda, B+\lambda$, and assume that $A$ and $B$ are invertible. We want to show that $A^{-1}-B^{-1}$ is compact.

The space $H^{-r}$ is dual to $H^{r}$ via the inner product in $H^{0}$. Specifically, we can consider $H^{s}$ as contained in $H^{t}$ for $s \geqq t$ in the obvious way. Then $H^{-r}$ is the completion of $H^{0}$ with respect to the norm (identical to the previously defined norm)

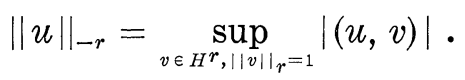

Similarly, define $W^{-r}$ as the completion of $H^{0}$ with respect to the norm 


$$
|u|_{-}=\sup _{v \in W r,\|v\|=1}|(u, v)| \text {. }
$$

Because of the definitions of the norms we have natural inclusions

$$
W^{r} \subset H^{r} \subset H^{0} \subset H^{-r} \subset W^{-r} \text {. }
$$

LEMma 3. A and $B$ have unique bounded extensions mapping $H^{r}$ onto $H^{-r}$ and $W^{r}$ onto $W^{-r}$ respectively.

Proof. For $u$ in the domain of $B$, (13) gives

$$
|B u|_{-}=\sup _{|v|=1}|(B u, v)|=\sup _{|v|=1}|b(u, v)| \leqq c_{4}|u| \text {. }
$$

Thus $B$ has a unique continuous extension $B_{1}$ mapping $W^{r}$ into $W^{-r}$. With our replacement of $B$ by $B+\lambda$ we have $c_{6}<0$ in (14), so $B_{1}$ has closed range in $W^{-r}$. The range includes $H^{0}$, hence is all of $W^{-r}$. Similarly $A$ has an extension $A_{1}$.

From now on we shall drop the subscripts on $A_{1}, B_{1}$ and consider $A, B$ as being defined either on their original domains or on $H^{r}, W^{r}$. Since $A^{*}$ maps $H^{m}$ onto $H^{0}$ [8], we can also, by duality, consider $A$ as mapping $H^{0}$ onto $H^{-m}$. With these conventions,

$$
\begin{aligned}
A^{-1}-B^{-1} & =A^{-1}(B-A) B^{-1}=A^{-1} q B^{-1} \\
& =\left(A^{-1} \rho\right)\left(\rho B^{-1}\right)-\left(A^{-1} \sigma\right)\left(\sigma B^{-1}\right)+i\left(A^{-1} \tau\right)\left(\tau B^{-1}\right)
\end{aligned}
$$

on $W^{-r}$. In fact $\left(\rho B^{-1}\right)$ is bounded from $W^{-r}$ to $H^{0}$ via $W^{r}$, while $A^{-1}$ is bounded from $H^{0}$ to itself via $H^{-m}$, by Lemma 1. Similarly for the other terms. We want to show that each term on the right in (16) is compact, but shall consider in detail only the first term.

Take $\varphi \in \mathscr{D}$ with $\varphi(x)=1,|x|<1 / 2$, and $\varphi(x)=0,|x|>1$. Let $\varphi_{t}(x)=\varphi\left(t^{-1} x\right), t>0$. We consider multiplication by $\varphi$ as an operator, also denoted by $\varphi$. Write

$$
A^{-1} \rho^{2} B^{-1}=A^{-1} \rho\left[\varphi_{t} \rho B^{-1}\right]+\left[A^{-1} \rho\left(1-\varphi_{t}\right)\right]_{\rho} B^{-1} .
$$

Lemma 1 and assumption (II) imply that the norm of the second term on the right as operator in $H^{0}$ goes to 0 as $t \rightarrow \infty$. Therefore in suffices to show that the first term on the right is compact. Assumption (I)' implies that $A^{-1} \rho \varphi_{t}$ is compact [9]. We shall show that in any case, $\varphi_{t_{1}} o B^{-1}$ is compact. In general, if $S$ is bounded from $W^{-r}$ or $H^{-r}$ to $H^{0}, \varphi S$ will not be compact in $H^{0}$. We must use particular properties of $B^{-1}$.

LEMMA 4. Let $S$ be a bounded operator in $H^{0}$ such that one of the following holds:

(a) $S$ is bounded from $L^{2}$ to $H^{s}$, some $s>0$; 
(b) $S$ is bounded from $H^{-s}$ to $L^{2}$, some $s>0$.

Suppose $\varphi \in \mathscr{D}$. Then in case (a), $\varphi S$ is compact in $H^{0}$, while in case (b), $S \varphi$ is compact in $H^{0}$.

Proof. This follows immediately from the well-known fact that $\varphi$ is compact as operator from $H^{s}$ to $H^{0}$ or from $H^{0}$ to $H^{-s}$.

We shall say that a bounded operator $S$ in $H^{0}$ is acceptable if it is of the form

$$
S=\left(\varphi_{1} S_{1} \psi_{1}\right)\left(\varphi_{2} S_{2} \psi_{2}\right) \cdots\left(\varphi_{k} S_{k} \psi_{k}\right)
$$

with $\varphi_{j}, \psi_{j} \in \mathscr{D}$ and each $S_{j}$ bounded in $H^{0}$. We say that $S$ in the form (17) is of weight:- $N$ if there are integers $N_{j} \geqq 0$ with $\Sigma N_{j} \geqq N$ such that for each $j, S_{j}$ is either bounded from $H^{0}$ to $H^{N_{j}}$ or from $H^{-N_{j}}$ to $H^{0}$.

If $\varphi \in \mathscr{D}, \varphi$ clearly maps $W^{r}$ and $W^{-r}$ into themselves. On $W^{-r}$ the commutator $\left[B^{-1}, \varphi\right]=B^{-1} \varphi-\varphi B^{-1}$ can be written

$$
\left[B^{-1}, \varphi\right]=B^{-1}[\varphi, B] B^{-1}=B^{-1}[\varphi, A] B^{-1}=B^{-1} C(\varphi) B^{-1},
$$

where $C(\varphi)=[\varphi, A]$ is a differential operator of order $<m$. It is easily seen that for $u, v \in \mathscr{D}$,

$$
(C(\varphi) u, v)=\Sigma\left(c_{\alpha \beta} D^{\beta} u, D^{\alpha} v\right)
$$

with the summation over $|\alpha| \leqq r,|\beta| \leqq r,|\alpha|+|\beta|<m$. The coefficients $c_{\alpha \beta}$ have bounded derivatives of order $\leqq \max (|\alpha|,|\beta|)$, with the bounds depending only on the bounds of derivatives of the $a_{\alpha \beta}$ and of $\varphi$. It follows readily from this, duality, and the equivalent form (2)' of the norm that

$$
\|C(\varphi) u\|_{k-m-1} \leqq c(\varphi)\|u\|_{k}, k=-1,0,1, \cdots, m,
$$

with

$$
c(\varphi)=c \sup _{\mid a, \leqq 2 m, x \in R^{n}}\left|D^{\alpha} \varphi(x)\right|,
$$

$c$ independent of $\varphi$. Note also that if $\psi \varphi=\varphi$, then

$$
C(\varphi)=\psi(\varphi)=C(\varphi) \psi \text {. }
$$

Let $s=r$ if $r$ is even, $s=r-1$ if $r$ is odd. Define $\Lambda^{ \pm}=G_{\mp s}$, where $G_{t}$ is as in $\S 2$. Then $\Lambda^{+}=(1-\Delta)^{s / 2}$ is a differential operator of order $s$.

Lemma 5. Suppose $\varphi, \psi \in \mathscr{D}$ and $N$ is an integer $\geqq 0$. Then $\varphi A^{-} C(r) B^{-1}$ can be written as a sum of acceptable terms of weight 
$-s-1$ plus a remainder term of the form $S T$ with $S$ acceptable of weight- $N$ and $T$ bounded in $H^{\circ}$.

Proof. Induce on $N$. For $N=0$, take $S=\varphi, T=A^{-} C\left(\psi^{\prime}\right) B^{-1}$. Otherwise take $\psi=\psi_{0}, \psi_{1}, \psi_{2} \in \mathscr{D}$ with $\psi_{j+1} \psi_{j}=\psi_{j}$. Set $C=C(\psi)$, $C_{1}=C\left(\psi_{1}\right), R=B^{-1}$. Then

$$
\begin{gathered}
\varphi \Lambda^{-} C R=\varphi \Lambda^{-} C \psi_{1} R \\
=\varphi \Lambda^{-} C R \psi_{1}+\varphi \Lambda^{-} C R C_{1} R, \\
R \psi_{1}=R \psi_{1} \Lambda^{+} \Lambda^{-} \psi_{2}=\left(R \psi_{1} \Lambda^{+} \psi_{2}\right)\left(\psi_{2} \Lambda^{-} \psi_{1}\right) .
\end{gathered}
$$

Now $\Lambda^{-} C R \psi_{1} \Lambda^{+}$is bounded from $H^{-1}$ to $H^{0}$ if $\mathrm{s}=r-1$ and from $H^{0}$ to $H^{1}$ if $s=r$, so the first term on the right in (20) can be expressed in the desired form (with no remainder). As for the second term,

$$
\begin{aligned}
\varphi \Lambda^{-} C R C_{1} R & =\varphi \Lambda^{-} C R \psi_{1} \Lambda^{+} \Lambda^{-} C_{1} R \\
& =\left[\varphi \Lambda^{-} C R \psi_{1} \Lambda^{+} \psi_{2}\right]\left[\psi_{2} \Lambda^{-} C_{1} R\right] .
\end{aligned}
$$

The first term in brackets is again acceptable of weight -1 , and we may apply the induction assumption to the second term.

Lemma 6. Suppose $\varphi \in \mathscr{D}$ and $N$ is an integer $\geqq 0$. Then $\varphi B^{-1}$ can be expressed as a sum of terms of the form $\varphi B^{-1} \psi^{+} S$ with $S$ acceptable of weight $\leqq-s$ and $\psi \in \mathscr{D}$, plus a remainder term of the form $\varphi B^{-1} \psi \Lambda^{+} S T$ with $S$ acceptable of weight $-N$ and $T$ bounded in $H^{0}$.

Proof. Take $\psi=\psi_{0}, \psi_{1}, \psi_{2} \in \mathscr{D}$ with $\psi \varphi=\varphi, \psi_{j+1} \psi_{j}=\psi_{j}$. Let $C=C(\psi), R=B^{-1}$. Then

$$
\varphi R=\varphi \psi R=\varphi R \psi+\varphi R C R .
$$

Now $\varphi R \psi$ can be expressed as in (21), while

$$
\varphi R C R=\varphi R \dot{\psi}_{1} C R=\left[\rho R \dot{\psi}_{1} \Lambda^{+} \psi_{2}\right]\left[\dot{\psi}_{2} \Lambda^{-} C R\right] .
$$

Applying Lemma 5 to the second term in brackets, we get what we want.

CoRollary. If $\varphi \in \mathscr{D}, \varphi \rho B^{-1}$ is a compact operator in $H^{0}$.

Proof. Lemma 6 shows that $\varphi \rho B^{-1}$ is a sum of terms of the form $\left[\rho \rho B^{-1} \psi \Lambda^{+}\right] S, \psi, \eta \in \mathscr{D}$ and $S$ bounded. Moreover, if $s>0$ then by Lemma $4 S$ is compact; if $s=0$ then $A^{+}=I$ and the term in brackets is compact, mapping $H^{-r}$ into $H^{0}$. 
The conclusion of the Corollary applies with $\rho$ replaced by $\sigma$ or $\tau$, so $A^{-1}-B^{-1}$ is compact. This proves Theorem 2 .

The following will be used in the proof of Theorem 3 .

Lemma 7. Suppose $\varphi \in \mathscr{D}$ and $N$ is an integer $\geqq 0$. Then $\varphi B^{-1}$ can be expressed as a sum of acceptable operators of weight $-m$, plus remainder terms of the form $S T, S$ acceptable of weight $-N$ and $T$ bounded in $H^{\circ}$.

Proof. When $s=r, B^{-1} \psi \Lambda^{+}$is bounded from $H^{0}$ to $H^{r}$ and this conclusion follows immediately from Lemma 6 . When $s=r-1$,

$$
\varphi B^{-1} \psi \Lambda^{+} \psi_{1}=\varphi B^{-1} \psi \Lambda^{+}(1-\Delta) \psi_{1}(1-\Delta)^{-1} \psi_{2} .
$$

Breaking up $\Lambda^{+}(1-\Delta)$ into monomial differential operators, we get a sum of terms $\varphi B^{-1} E_{1} \psi_{1} \psi_{2} E_{2}(1-\Delta)^{-1} \psi_{2}$, with $E_{1}, E_{2}$ differential operators of orders $\leqq r$ and $\leqq 1$ respectively. These terms are then acceptable of weight $(-r)+(-1)$. Thus again the conclusion follows from Lemma 6.

Lemma 8. Suppose $\varphi \in \mathscr{D}$ and $N$ an integer $\geqq 0$. Then $A^{-1} \varphi$ can be expressed as a sum of terms of the form $S A^{-1} \varphi$ with $S$ acceptable of weight 0 , plus a remainder term of the form $T S A^{-1} P$ with $S$ acceptable of weight $-N$ and $T$ bounded in $H^{\circ}$.

Proof. Take $\psi_{0}, \psi_{1}, \psi_{2}, \cdots$ in $\mathscr{D}$ with $\psi_{2} \varphi=\varphi, \psi_{j+1} \psi_{j}=\psi_{j}$. Let $R=A^{-1}, C_{j}=\left[\varphi_{j}, A\right]$. Then

$$
\begin{aligned}
R \varphi & =\psi_{0} R-R C_{0} R \varphi \\
& =\psi_{0} R \varphi-\psi_{1} R C_{0} R \varphi+R C_{1} R C_{0} R \varphi . \\
& =\cdots .
\end{aligned}
$$

Now $R C_{j}$ maps $H^{-1}$ to $H^{0}$, so $\psi_{j+2} R C_{j} \psi_{j+1}$ is acceptable of weight -1 . Continuing as above, we get acceptable terms of the form we want, plus a remainder term

$$
\pm R C_{N}\left[R C_{N-1} R \cdots R C_{0}\right] R \varphi .
$$

Take $\pm R C_{N}$ as $T$ and write the term in brackets as a product of terms $\psi_{j+2} R C_{j} \psi_{j+1}$.

4. Proof of Theorems 3 and 4. Once again, given $\psi \in C_{c}^{\infty}$, let $\psi_{t}(x)=\psi\left(t^{-1} x\right)$. It follows from the equivalent form $(2)^{\prime}$ of the norm that for integer $l \geqq 0$, 


$$
\|\varphi u\|_{l} \leqq c_{l} \sup _{|\alpha| \leqq l, x \in R^{n}}\left|D^{\alpha} \varphi(x)\right|\|u\|_{l} .
$$

Thus $\psi_{t}$ is bounded uniformly with respect to $t, t \geqq 1$, as a map from $H^{l}$ to itself. By duality the same is true for integers $l<0$.

Lemma 9. Suppose $\varphi \in \mathscr{D}$ and $\varphi_{t}(x)=\varphi\left(t^{-1} x\right)$. For each integer $l \geqq 0$, each $t \geqq 1$, and each integer $j \geqq 1$ there is a subspace $H_{j}$ of $H^{0}$ having codimension $j-1$, such that

$$
\left\|\varphi_{t} u\right\|_{-l} \leqq c j^{-l / n} \cdot t^{l}\|u\|_{0}, u \in H_{j} .
$$

The constant $c=c(l)$ can be chosen independent of $j$ and $t, t \geqq 1$.

Proof. Let $\Omega_{t}$ be the cube $\left\{x|| x_{k} \mid<t, k=1, \cdots, n\right\}$. We may assume that $\varphi(x)=0$ outside $\Omega_{1}$. Choose $\psi \in \mathscr{D}$ with $\int \psi(\xi) d \xi=(2 \pi)^{n / 2}$, and let $\eta$ be the inverse Fourier transform of $\psi$. Then $\eta(0)=1$. Replacing $\psi$ by $\varepsilon^{-n} \psi\left(\varepsilon^{-1} x\right)$ for small enough $\varepsilon>0$, we may assume both that $|\eta(x)| \geqq \frac{1}{2}$ on $\Omega_{1}$ and that $\psi$ vanishes outside $\Omega_{1}$. Let $\eta_{t}(x)=\eta\left(t^{-1} x\right)$. For a given $t \geqq 1$ and $\alpha$ an $n$-tuple of integers, let $\alpha \cdot x=\Sigma \alpha_{k} x_{k},|\alpha|=\Sigma\left|\alpha_{k}\right|$, and set

$$
\begin{aligned}
e_{\alpha}(x) & =(2 t)^{-n / 2} \exp \left(t^{-1} \pi i \alpha \cdot x\right), x \in \Omega_{t}, \\
& =0, \notin \Omega_{t} ; \\
f_{\alpha}(x) & =(2 t)^{-n / 2} \eta_{t}(x) \exp \left(t^{-1} \pi i \alpha \cdot x\right), \text { all } x .
\end{aligned}
$$

Then

$$
\widehat{f}_{\alpha}(\xi)=(t / 2)^{n / 2} \psi(t \xi+\pi i \alpha) .
$$

It follows that $\hat{f}_{\alpha} \hat{f}_{\beta} \equiv 0$ for $\alpha \neq \beta$, so the $f_{\alpha}$ are orthogonal as elements of $H^{-l}$. Let $\alpha(1), \alpha(2), \cdots$, be an enumeration of the $n$-tuples $\alpha$ with $|\alpha(j)| \leqq|\alpha(j+1)|$. Let $e_{k}=e_{\alpha(k)}, f_{k}=f_{\alpha^{\prime}(k)}$. It follows from (24) and (2) that

$$
\left\|f_{k}\right\|_{-l} \leqq c_{0} t^{l}|\alpha(k)|^{-l} \leqq c_{1} t_{k}^{l-l / n}
$$

for $|\alpha|>0$, with $c_{1}$ independent of $k$ and $t, t \geqq 1$.

Let $\mathrm{H}_{j}$ be the orthogonal complement of $\left\{e_{1}, \epsilon_{2}, \cdots, e_{j_{-1}}\right\}$ in $H^{0}$. The $e_{k}$ are an orthonormal basis for $L^{2}\left(\Omega_{t}\right)$, so for any $u \in H^{0}$,

$$
u=\Sigma a_{k} e_{k} \text { on } \Omega_{t}, \Sigma\left|a_{k}\right|^{2} \leqq\|u\|_{0}^{2},
$$

with $a_{k}=\left(u, e_{k}\right)$. If $u \in H_{j}$,

$$
\varphi_{t} u=\varphi_{t} \sum_{k \geq j} a_{k} e_{k}=\left(\varphi_{t} \eta_{t}^{-1}\right) \sum_{k \geqq j} a_{k} f_{k} .
$$

Since the $f_{k}$ are orthogonal in $H^{-l},(25),(26),(27)$ and the remarks 
preceding the lemma give (23), with $c$ independent of $j$ and $t, t \geqq 1$.

Lemma 10. Suppose $S$ is bounded in $H^{0}$ and either

or

(a) $\quad\|S u\|_{0} \leqq M\|u\|_{-l}$, all $u \in H^{0}$

(b) $\|S u\|_{l} \leqq M\|u\|_{0}$, all $u \in H^{0}$,

where $l$ is a positive integer. Suppose $\varphi \in \mathscr{D}$ and $\varphi_{t}(x)=\varphi\left(t^{-1} x\right)$. Set $T_{t}=S \varphi_{t}$ in case (a), $T_{t}=\varphi_{t} S$ in case (b). Then the characteristic numbers satisfy

$$
\mu_{j}\left(T_{t}\right) \leqq c M_{j}^{-l / n} \cdot t^{l},
$$

where $c$ is independent of $j$ and $t, t \geqq 1$.

Proof. Suppose (a). Given $t \geqq 1$, let $H_{j}$ be as in Lemma 9 and apply (4), (23), and (a). Case (b) follows from (a) and the fact that $\mu_{j}(T)=\mu_{j}\left(T^{*}\right)$.

Now consider Theorem 3. Again we may assume $\lambda=0$ and look at $A^{-1}-B^{-1}, p$ a positive integer. On $W^{-r}$,

$$
\begin{aligned}
A^{-p}-B^{-p} & =\sum_{j=0}^{p-1} A^{j+1-p}\left[A^{-1}-B^{-1}\right] B^{-j} \\
& =\sum_{j=0}^{p-1} A^{j-p} q B^{-j-1} .
\end{aligned}
$$

Once again take $\varphi \in \mathscr{D}$ with $\varphi(x)=1,|x|<\frac{1}{2}$, and $\varphi(x)=0,|x|>1$, and set $\varphi_{t}(x)=\varphi\left(t^{-1} x\right)$. Repeated applications of Lemmas 6,7 and 8 show that

$$
A^{j-1} \varphi_{t} \rho^{2} \varphi_{t} B^{1-j}
$$

can be written as a sum of terms of the form

$$
T_{1} S_{1}\left(A^{-1} \rho\right) \varphi_{t}^{2}\left(B^{-1}\right) S_{2} T_{2} \text {. }
$$

Here $\psi, \eta \in \mathscr{D}, T_{1}$ and $T_{2}$ are bounded in $H^{0}$, and $S_{1}$ and $S_{2}$ are acceptable of weights $s_{1}$ and $s_{2}$ with

$$
s_{1}=(p-j-1) m, s_{2}=j m .
$$

Furthermore the numbers of summands and factors are independent of $t$, and the operator norms are bounded independent of $t \geqq 1$. Recall also that $\left(A^{-1} \rho\right)$ is bounded in $H^{0}$ and $\rho B^{-1}$ is bounded from $H^{-r}$ to $H^{0}$.

In general, characteristic numbers satisfy

$$
\begin{gathered}
\mu_{j_{k+1}}\left(S_{1} S_{2} \cdots S_{k}\right) \leqq \mu_{j+1}\left(S_{1}\right) \mu_{j+1}\left(S_{2}\right) \cdots \mu_{j+1}\left(S_{k}\right), \\
\mu_{j_{k+1}}\left(S_{1}+\cdots+S_{k}\right) \leqq \mu_{j+1}\left(S_{1}\right)+\cdots+\mu_{j+1}\left(S_{k}\right)
\end{gathered}
$$




\section{[3], Corollary X1.9.3. Also clearly $\mu_{j}(S) \leqq\|S\|$.}

Now write each term on the right in (29) as a sum of a term with $q \varphi_{t}^{2}$ and a term with $q\left(1-\varphi_{t}^{2}\right)$. Grouping the former and latter terms together we get

$$
A^{-p}-B^{-p}=D_{t}+E_{t} .
$$

Our assumptions (III) $)_{a}$ or (III) $)_{a}^{\prime}$ together with Lemma 1 and the fact that $\left(1-\varphi_{t}^{2}\right)=0$ for $|x|<t / 2$ give

$$
\left\|E_{t}\right\|=0\left(t^{-a}\right),
$$

where again the norm is the operator norm in $H^{0}$. In case (III) $)_{a}^{\prime}$ this comes from (8) applied to $A^{-1} q\left(1-\varphi_{t}^{2}\right)^{1 / 2}$ and (7) applied to $\left(1-\varphi_{t}^{2}\right)^{1 / 2} q B^{-1}$, while in case (III) a we apply (7) to $A^{-1} \rho\left(1-\varphi_{t}^{2}\right)$ and treat the terms with $\sigma$ and $\tau$ as in case (III)'.

Finally, (28) - (35) imply

$$
\mu_{j}\left(A^{-p}-B^{-p}\right) \leqq c\left(t^{-a}+t^{l} \cdot j^{-l / n}\right),
$$

with $c$ independent of $j$ and $t, t \geqq 1$, and with

$$
l=(p-j-1) m+r+j m=\left(p-\frac{1}{2}\right) m ;
$$

the extra $r$ coming from $\varphi_{t} q B^{-1}$. Now let $t=j^{b}$ with $b=l / n(a+l)$. Then (36) becomes

$$
\mu_{j}\left(A^{-1}-B^{-p}\right)=0\left(j^{-\nu}\right), \nu=a l / n(a+l) .
$$

As $p \rightarrow \infty, \nu=\nu(p) \rightarrow a / n$. This proves Theorem 3 .

Finally, under the assumptions of Theorem 4,

$$
\mu_{j}=\mu_{j}\left((A+\lambda)^{-p}-(B+\lambda)^{-p}\right)=0\left(j^{-1-\varepsilon}\right)
$$

for $\operatorname{Re} \lambda \geqq \lambda_{0}$, large enough $p$, and small enough $\varepsilon>0$. Thus $\Sigma \mu_{j}<\infty$, and $(A+\lambda)^{-p}-(B+\lambda)^{-p}$ is a trace class operator. The conclusions of Theorem 4 now follow from results of Kuroda and Kato; see [5], Theorem 4.12 and Remark 4.13 .

\section{REFERENCES}

1. E. Balslev, The essential spectrum of self-adjoint differential operators in $L^{2}\left(R_{n}\right)$, Math. Scand., 19 (1966), 193-210.

2. M. S. Birman, On the spectrum of singular boundary value problems, Mat. Sb., 97 (1961), 125-174; Amer. Math. Soc. Translations (2), vol. 53 (1966), 23-80.

3. N. Dunford and J. T. Schwartz, Linear Operators, Vol. 2, 1963, Interscience.

4. J. S. Howland, On the essential spectrum of Schrodinger operators with singular potentials, Pacific J. Math., 25 (1968), 533-542.

5. T. Kato, Perturbation Theory for Linear Operators, 1966, Springer-Verlag.

6. P. A. Rejto, On the essential spectrum of the hydrogen energy and related operators, Pacific J. Math., 19 (1966), 109-140. 
7. M. Schechter, On the essential spectrum of an arbitrary operator, J. Math. Anal. Appl., 13 (1966), 205-215.

8. - On the invariance of the essential spectrum of an arbitrary operator, II, Richerche Mat., 16 (1967), 3-26.

9. - On the invariance of the essential spectrum of an arbitrary operator, III, Proc. Camb. Phil. Soc., 64 (1968), 975-984.

Received August 4, 1969 and in revised form September 11, 1970. Research supported ] in part by the Army Research Office, Durham.

UNIVERSITY OF CHICAGO 


\section{PACIFIC JOURNAL OF MATHEMATICS}

\section{EDITORS}

H. SAMELSON

Stanford University

Stanford, California 94305

C. R. Новву

University of Washington

Seattle, Washington 98105
J. DUGundJI

Department of Mathematics

University of Southern California

Los Angeles, California 90007

RICHARD ARENS

University of California

Los Angeles, California 90024

\section{ASSOCIATE EDITORS}
E. F. BECKENBACH
B. H. NeumanN
F. WOLF
K. YOSHIDA

\section{SUPPORTING INSTITUTIONS}
UNIVERSITY OF BRITISH COLUMBIA
CALIFORNIA INSTITUTE OF TECHNOLOGY
UNIVERSITY OF CALIFORNIA
MONTANA STATE UNIVERSITY
UNIVERSITY OF NEVADA
NEW MEXICO STATE UNIVERSITY
OREGON STATE UNIVERSITY
UNIVERSITY OF OREGON
OSAKA UNIVERSITY
UNIVERSITY OF SOUTHERN CALIFORNIA
STANFORD UNIVERSITY
UNIVERSITY OF TOKYO
UNIVERSITY OF UTAH
WASHINGTON STATE UNIVERSITY
UNIVERSITY OF WASHINGTON
AMERICAN MATHEMATICAL SOCIETY CHEVRON RESEARCH CORPORATION TRW SYSTEMS
NAVAL WEAPONS CENTER 


\section{Pacific Journal of Mathematics}

\section{Vol. 37, No. $1 \quad$ January, 1971}

Gregory Frank Bachelis and Haskell Paul Rosenthal, On unconditionally

converging series and biorthogonal systems in a Banach space .........

Richard William Beals, On spectral theory and scattering for elliptic

operators with singular potentials .........................

J. Lennart (John) Berggren, Solvable and supersolvable groups in which every element is conjugate to its inverse ........................ 21

Lindsay Nathan Childs, On covering spaces and Galois extensions ..........

William Jay Davis, David William Dean and Ivan Singer, Multipliers and

unconditional convergence of biorthogonal expansions..............

Leroy John Derr, Triangular matrices with the isoclinal property ............

Paul Erdős, Robert James McEliece and Herbert Taylor, Ramsey bounds for

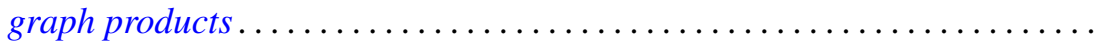

Edward Graham Evans, Jr., On epimorphisms to finitely generated

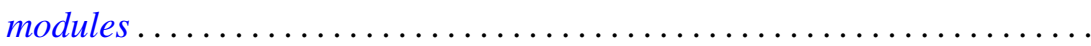

Hector O. Fattorini, The abstract Goursat problem ................. 51

Robert Dutton Fray and David Paul Roselle, Weighted lattice paths .........

Thomas L. Goulding and Augusto H. Ortiz, Structure of semiprime $(p, q)$

radicals ...........................................

E. W. Johnson and J. P. Lediaev, Structure of Noether lattices with join-principal maximal elements ....

David Samuel Kinderlehrer, The regularity of minimal surfaces defined over

slit domains

Alistair H. Lachlan, The transcendental rank of a theory. .

Frank David Lesley, Differentiability of minimal surfaces at the boundary ...

Wolfgang Liebert, Characterization of the endomorphism rings of divisible torsion modules and reduced complete torsion-free modules over complete discrete valuation rings....

Lawrence Carlton Moore, Strictly increasing Riesz norms.

Raymond Moos Redheffer, An inequality for the Hilbert transform ...

James Ted Rogers Jr., Mapping solenoids onto strongly self-entwined,

circle-like continua..........................

Sherman K. Stein, B-sets and planar maps ................... 217

Darrell R. Turnidge, Torsion theories and rings of quotients of Morita

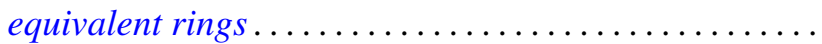

Fred Ustina, The Hausdorff means of double Fourier series and the principle of localization ................................

Stanley Joseph Wertheimer, Quasi-compactness and decompositions for arbitrary relations.

Howard Henry Wicke and John Mays Worrell Jr., On the open continuous images of paracompact $\check{C}$ ech complete spaces... 